\title{
Extra-ocular muscle MRI in genetically-defined mitochondrial disease
}

\author{
Robert D. S. Pitceathly ${ }^{1}$ - Jasper M. Morrow ${ }^{1}$ Christopher D. J. Sinclair ${ }^{1,2}$ • \\ Cathy Woodward ${ }^{3}$ - Mary G. Sweeney ${ }^{3}$ - Shamima Rahman ${ }^{1,4}$ • Gordon T. Plant ${ }^{5,6}$. \\ Nadeem Ali $^{5,6}$ - Fion Bremner ${ }^{5}$ - Indran Davagnanam ${ }^{7}$ - Tarek A. Yousry ${ }^{1,2}$. \\ Michael G. Hanna ${ }^{1}$ • John S. Thornton ${ }^{1,2}$
}

Received: 7 January 2015 /Revised: 28 March 2015 / Accepted: 14 April 2015 / Published online: 21 May 2015

(C) The Author(s) 2015. This article is published with open access at Springerlink.com

\begin{abstract}
Objectives Conventional and quantitative MRI was performed in patients with chronic progressive external ophthalmoplegia (CPEO), a common manifestation of mitochondrial disease, to characterise MRI findings in the extraocular muscles (EOMs) and investigate whether quantitative MRI provides clinically relevant measures of disease.

Methods Patients with CPEO due to single mitochondrial DNA deletions were compared with controls. Range of eye movement (ROEM) measurements, peri-orbital 3 T MRI T1weighted (T1w) and short-tau-inversion-recovery (STIR) images, and $\mathrm{T} 2$ relaxation time maps were obtained. Blinded observers graded muscle atrophy and T1w/STIR hyperintensity. Cross-sectional areas and EOM mean T2s were recorded and correlated with clinical parameters.
\end{abstract}

Robert D. S. Pitceathly

r.pitceathly@ucl.ac.uk

1 MRC Centre for Neuromuscular Diseases, UCL Institute of Neurology and National Hospital for Neurology and Neurosurgery, Queen Square, London WC1N 3BG, UK

2 Neuroradiological Academic Unit, Department of Brain Repair and Rehabilitation, UCL Institute of Neurology, London WC1N 3BG, UK

3 Neurogenetics Unit, National Hospital for Neurology and Neurosurgery, Queen Square, London WC1N 3BG, UK

4 Mitochondrial Research Group, Clinical and Molecular Genetics Unit, UCL Institute of Child Health, London WC1N 1EH, UK

5 Department of Neuro-ophthalmology, National Hospital for Neurology and Neurosurgery, London WC1N 3BG, UK

6 Department of Neuro-ophthalmology, Moorfields Eye Hospital, London EC1V 2PD, UK

7 The Lysholm Department of Neuroradiology, National Hospital for Neurology and Neurosurgery, London WC1N 3BG, UK
Results Nine patients and nine healthy controls were examined. Patients had reduced ROEM (patients $13.3^{\circ}$, controls $49.3^{\circ}, \mathrm{p}<0.001$ ), greater mean atrophy score and increased $\mathrm{T} 1 \mathrm{w}$ hyperintensities. EOM mean cross-sectional area was $43 \%$ of controls and mean T2s were prolonged (patients $75.6 \pm 7.0 \mathrm{~ms}$, controls $55.2 \pm 4.1 \mathrm{~ms}, \mathrm{p}<0.001)$. ROEM correlated negatively with EOM T2 (rho $=-0.89, \mathrm{p}<0.01)$, whilst cross-sectional area failed to correlate with any clinical measures.

Conclusions MRI demonstrates EOM atrophy, characteristic signal changes and prolonged T2 in CPEO. Correlation between elevated EOM T2 and ROEM impairment represents a potential measure of disease severity that warrants further evaluation.

Key Points

- Chronic progressive external ophthalmoplegia is a common clinical manifestation of mitochondrial disease.

- Existing extra-ocular muscle MRI data in CPEO reports variable radiological findings.

- MRI confirmed EOM atrophy and characteristic signal changes in CPEO.

- EOM T2 was significantly elevated in CPEO and correlated negatively with ocular movements.

- EOM T2 represents a potential quantitative measure of disease severity in CPEO.

Keywords Magnetic resonance imaging · Mitochondrial diseases · Mitochondrial DNA - Chronic progressive external ophthalmoplegia $\cdot$ Kearns-Sayre syndrome
Abbreviations
CPEO Chronic progressive external ophthalmoplegia
EOM(s) Extra-ocular muscle(s)
IO Inferior oblique 


$\begin{array}{ll}\text { IR } & \text { Inferior rectus } \\ \text { KSS } & \text { Kearn-Sayre syndrome } \\ \text { LR } & \text { Lateral rectus } \\ \text { MR } & \text { Medial rectus } \\ \text { mtDNA } & \text { Mitochondrial DNA } \\ \text { ROEM } & \text { Range of eye movement } \\ \text { ROI } & \text { Region of interest } \\ \text { STIR } & \text { Short-tau-inversion-recovery } \\ \text { SO } & \text { Superior oblique } \\ \text { SR } & \text { Superior rectus }\end{array}$

\section{Introduction}

There are currently no effective treatments for mitochondrial diseases [1,2]. One major obstacle to clinical trials is a lack of reliable, non-invasive, reproducible biomarkers against which disease progression and treatment response can be measured. Chronic progressive external ophthalmoplegia (CPEO), a common clinical manifestation of mitochondrial disease, is characterised by gradually progressive reduction in the range of eye movements (ROEM). It is usually sporadic, as occurs with single large-scale deletions of mitochondrial DNA (mtDNA) which have a reported point prevalence for clinically affected adults of 1.17 in 100,000 people [3]. However, it can also be inherited in a maternal or Mendelian pattern depending on whether the causative genetic defect resides in the mitochondrial or nuclear genome. Imaging studies have reported extra-ocular muscle (EOM) atrophy, sometimes associated with EOM signal change, as a feature of CPEO. However, investigations have been, for the most part, limited to small, genetically undefined patient series with rather variable radiological findings [4-7]. For instance, CPEO has been associated with normal or near normal volumes despite clinically severe ophthalmoplegia on orbital T1-weighted (T1w) MRI [6], whilst small EOMs are also reported using the same imaging modality $[4,5]$. Furthermore, EOM MRI signal abnormality in patients with CPEO, in the absence of orbital or EOM inflammation, has previously been proposed as a possible discriminator from other conditions which cause ophthalmoparesis [6]. However, this finding has not been replicated in independent patient cohorts with CPEO.

Our purpose was to clarify the radiological appearance of the EOMs in comparison with matched healthy individuals studied concurrently and, by correlation with clinical measures, investigate the feasibility and validity of quantitative MRI indices as functionally relevant markers of mitochondrial disease severity. We therefore conducted a peri-orbital MRI study in a genetically homogenous CPEO patient group with single deletions of mtDNA. We aimed to define in patients with CPEO the qualitative appearance of EOM on T1w and short-tau inversion recovery (STIR) MRI images, EOM spinspin relaxation times (T2s) and cross-sectional areas, in comparison with controls, and to correlate MRI findings with clinical measures including ROEM.

\section{Materials and methods}

\section{Patient and healthy control recruitment criteria}

Nine adult patients referred to the NHS Specialised Servicesfunded Mitochondrial Diagnostic Centre in London for investigation of CPEO and found to harbour a single, sporadically occurring, mtDNA deletion in skeletal muscle, and nine ageand gender-matched healthy controls were recruited. The mean age of the patients was 33 years (range: 19-52 years) with a mean age at symptom onset of 16 years (range: 12 32 years). Four patients with a clinical diagnosis of KearnsSayre syndrome (KSS) had a mean age at onset of 12 years (range: 6-17 years). The remaining patients with CPEO had a mean age of onset of 20 years (range: $14-32$ years). The mean age of the controls was 33 years (range: $26-43$ years).

Institutional Research Ethics Committee approval for the study, and accordingly individual subjects' consent, was obtained.

\section{Clinical assessment of ocular movements}

EOM function was quantified in terms of ROEM by measuring ocular excursions (ductions) for each eye on the Goldmann perimeter using, as in a previous CPEO study [8], the modified uniocular field of fixation technique [9]. This isolates the action of each individual muscle by recording excursions only along the axes corresponding to that muscle's primary field of action. These are plotted on standard Goldmann charts with pre-printed testing axes. For the right eye the axes are: $0^{\circ}$ (lateral rectus, LR); $67^{\circ}$ (superior rectus, $\mathrm{SR}$ ); $141^{\circ}$ (inferior oblique, IO); $180^{\circ}$ (medial rectus, MR); $216^{\circ}$ (superior oblique, SO); and $293^{\circ}$ (inferior rectus, IR). The left eye uses a chart which is the mirror image of the right eye chart. Testing was performed uniocularly with chin and head fixation and excursions repeated to ensure measurement consistency within $5^{\circ}$.

\section{Magnetic resonance imaging protocol}

Imaging was performed at $3 \mathrm{~T}$ (TIM Trio, Siemens, Erlangen, Germany) with a 32-channel receiver head coil. Subjects were examined supine and headfirst and asked to keep their eyes closed for the duration of each scan. Coronal images were acquired with a field of view (FOV) of $162 \times 200 \mathrm{~mm}$. Turbo-spin echo (TSE) T1w images were acquired with time to repetition (TR)/time to echo (TE) $=575 / 7.6 \mathrm{~ms}, 20 \times 2-\mathrm{mm}$ slices, slice gap $1 \mathrm{~mm}$, matrix $312 \times 384$. STIR images were acquired with TR/TE/time to inversion $=6760 / 8 / 220 \mathrm{~ms}$, 
matrix $312 \times 384$ and $20 \times 2$-mm slices, slice gap $1 \mathrm{~mm}$. T2 was measured with a multiple spin-echo sequence $(\mathrm{TR}=$ $4060 \mathrm{~ms}, 22$ spin-echoes with TEs 8.6-189.2 ms and an echo spacing of $8.6 \mathrm{~ms}$, matrix $208 \times 256,12 \times 3-\mathrm{mm}$ slices, $0.3-\mathrm{mm}$ gap, $180^{\circ}$ refocusing pulses). Total imaging time was less than $20 \mathrm{~min}$.

\section{Qualitative magnetic resonance imaging evaluation}

All MRI analysis was performed by observers blinded to the subject clinical details, including patient versus control status. The coronal T1w and STIR orbital images were systematically reviewed by two experienced neuroradiologists (I.D. and T.Y.). The individual characteristics of all 12 EOMs were reviewed sequentially in all subjects and the consensus opinion was recorded. A scoring system was applied to capture the following qualitative features: degree of atrophy (normal $=0$, mild $=1$, marked $=2$ ); STIR hyperintensity (normal $=0$, hyperintense $=1$ ); and presence or absence of $\mathrm{T} 1 \mathrm{w}$ hyperintensity foci with anatomically coincident STIR hypointensity (presumed to represent fatty infiltration), taking account of the intra-EOM pattern: the intrinsic foci were observed to either be of approximately equal diameter in all dimensions in the coronal plane, termed 'dots', or elongated in one dimension (invariably in-line with the longest dimension of the extraocular muscle in the coronal section), termed 'streaks'. Where both dots and streaks were observed in different sections through a single EOM, the convention adopted was to record the muscle as demonstrating an intrinsic 'streak' signal. The qualitative scores were tabulated for both healthy controls and CPEO patients.

\section{Measurement of cross-sectional area}

Cross-sectional areas of the five EOMs bilaterally (excluding IO) were obtained from the coronal T1w images by outlining regions of interest (ROI) around the outer border of each muscle, one slice behind the posterior border of the globe, using the FSLView software (FMRIB, Oxford, UK), and the crosssectional area of each was recorded. All measurements were performed by a single observer (R.D.S.P) and reviewed by a second observer (I.D.) for quality control purposes.

\section{Quantitative magnetic resonance analysis}

A single observer (J.M.) defined additional ROIs on the TE= $8.6 \mathrm{~ms}$ multi-echo image of the first coronal slice posterior to the globe, for the four rectus muscles bilaterally using the ITK-SNAP software [10]. Only voxels wholly within the muscle border were included to avoid partial volume errors arising from pixels containing both muscle and orbital fat. The small size of the superior oblique EOM prevented reliable ROI placement and this EOM was therefore omitted from the analysis. The mean ROI signal intensities were calculated for each TE image and fitted to a mono-exponential decay function to obtain the intercept, T2 decay constant and a DC offset using the Levenberg-Marquardt algorithm implemented in Wolfram Mathematica (Champaign, IL, USA).

\section{Statistical methods}

As the different analyses included different muscle subgroups (all 12 muscles for ROEM analyses, ten muscles for a crosssectional area, eight muscles for T2 measurement) when overall means were calculated only the common set of muscles were included, left and right: SR, IR, LR and MR. Mean values in patient and control groups, reported with their respective standard deviations, were compared using the twotailed Student's t-test. The proportion of muscles in each group with T1w hyperintensity was compared with Pearson's $\chi^{2}$ test. Correlations between MRI measures were analysed using the Spearman rank correlation (rho). All statistical analyses were performed using IBM SPSS Version 20.

\section{Results}

\section{Clinical description}

The demographic, clinical and quantitative MRI findings for the patient group are summarised in Table 1, and for the control group in Table 2.

\section{Clinical assessment of ocular movements}

The overall mean ROEM was reduced by $73 \%$ in the patient group compared with controls (patients $13.3^{\circ}$, controls $49.3^{\circ}$, $\mathrm{p}<0.001$; Table 3 ), with a consistent reduction in individual EOM ROEMs (Fig. 1A). There was no overlap between patient and control range of movement values for any muscle. There was no significant quantitative difference in ROEM detected between the right and left orbit in patients or controls, or between the CPEO and KSS subgroups, when muscles were compared both individually and collectively.

\section{Magnetic resonance imaging analysis}

On qualitative assessment, the mean atrophy score in patients was $1.96 \pm 0.11$, significantly higher than in controls $(0.65 \pm$ $0.59, \mathrm{p}<0.001$ ). Only two participants (both patients with CPEO) had focal STIR hyperintensity within EOMs, in 2/12 and 4/12 EOMs, respectively. Focal T1w hyperintensity was more common in patients than controls $(72 / 108$ muscles in patients, $49 / 108$ muscles in controls, $\chi^{2}=0.002$ ). In patients this more commonly manifested as a streak (63 muscles) than 
Table 1 Demographic, clinical and magnetic resonance imaging findings in patients with chronic progressive external ophthalmoplegia related to a single mitochondrial DNA deletion

\begin{tabular}{|c|c|c|c|c|c|c|c|}
\hline Gender & $\begin{array}{l}\text { Age } \\
\text { (years) }\end{array}$ & $\begin{array}{l}\text { Clinical } \\
\text { diagnosis }\end{array}$ & $\begin{array}{l}\text { Age of } \\
\text { onset (years) }\end{array}$ & Clinical characteristics & $\begin{array}{l}\text { Mean } \\
\text { ROEM } \\
\text { (degrees) }\end{array}$ & $\begin{array}{l}\text { Mean } \\
\text { T2 (ms) }\end{array}$ & $\begin{array}{l}\text { Mean } \\
\text { EOM area } \\
\left(\mathrm{mm}^{2}\right)\end{array}$ \\
\hline $\mathrm{F}$ & 19 & KSS & 12 & $\begin{array}{l}\text { PEO; ptosis; pigmentary retinopathy; proximal muscle weakness; } \\
\text { ataxia; SNHL; fatigue; short stature; low BMI; scoliosis }\end{array}$ & 17.3 & 80.5 & 12.3 \\
\hline M & 28 & CPEO & 16 & PEO; ptosis; fatigue & 10.6 & 70.1 & 20.5 \\
\hline M & 28 & CPEO & 14 & PEO; ptosis & 20.9 & 67.8 & 20.4 \\
\hline $\mathrm{F}$ & 28 & KSS & 12 & $\begin{array}{l}\text { PEO; ptosis; pigmentary retinopathy; fatigue; dysphagia; facial, } \\
\text { truncal, and proximal muscle weakness; migraine; reduced ET; } \\
\text { pain; cramps; migraine; SVT }\end{array}$ & 8.8 & 85.3 & 12.7 \\
\hline $\mathrm{F}$ & 29 & KSS & 6 & $\begin{array}{l}\text { PEO; ptosis; fatigue; learning difficulties; SNHL; pigmentary } \\
\text { retinopathy; DM; hypertension }\end{array}$ & 25.8 & 69.5 & 15.2 \\
\hline $\mathrm{F}$ & 35 & CPEO & 14 & PEO; ptosis; fatigue; low BMI; proximal muscle weakness; pain & 3.2 & 79.9 & 15.1 \\
\hline $\mathrm{F}$ & 36 & KSS & 17 & $\begin{array}{l}\text { PEO; epilepsy; migraine; pigmentary retinopathy; proximal } \\
\text { muscle weakness }\end{array}$ & 3.1 & 85.7 & 18.6 \\
\hline M & 45 & CPEO & 24 & PEO; ptosis; fungal keratitis (blind in RE) & NA & 83.2 & 15.4 \\
\hline M & 52 & CPEO & 32 & PEO; ptosis & 16.6 & 78.0 & 21.7 \\
\hline
\end{tabular}

Mean values are across left and right lateral rectus, inferior rectus, medial rectus, and superior rectus

$B M I$ body mass index, $C P E O$ chronic external progressive ophthalmoplegia, DM diabetes mellitus, EOM extra-ocular muscle, ET exercise tolerance, KSS Kearns-Sayre syndrome, $N A$ not available, $R E$ right eye, ROEM range of eye movement, $S N H L$ sensorineural hearing loss, $S V T$ supraventricular tachycardia

a dot (nine muscles), whereas in the controls the counts were 25 and 24, respectively (Fig. 2A, 2B, 2C, 2D; Table 4).

The mean EOM cross-sectional area in patients was $43 \%$ lower than for controls (patient $19.2 \pm 3.9 \mathrm{~mm}^{2}$, control $33.6 \pm$ $3.1 \mathrm{~mm}^{2}, \mathrm{p}<0.001$; Fig. 2E and F; Table 3). Muscle T2s were greater in the patient group than controls $(75.6 \pm 7.0 \mathrm{~ms}$, vs. 55.2 $\pm 4.1 \mathrm{~ms}$ respectively, $\mathrm{p}<0.001$; Fig. $2 \mathrm{G}$, h, J; Table 3). Consistent individual EOM cross-sectional area and T2 differences were observed (Fig. 1B and C). Quantitative differences in mean ROEM, EOM cross-sectional area and T2 were consistently significant when each muscle was assessed

Table 2 Demographic, clinical and magnetic resonance imaging findings in healthy controls

\begin{tabular}{lllll}
\hline Gender & Age (years) & $\begin{array}{l}\text { Mean ROEM } \\
\text { (degrees) }\end{array}$ & $\begin{array}{l}\text { Mean } \\
\text { T2 }(\mathrm{ms})\end{array}$ & $\begin{array}{l}\text { Mean EOM } \\
\text { area }\left(\mathrm{mm}^{2}\right)\end{array}$ \\
\hline F & 26 & 51.5 & 46.9 & 32.3 \\
M & 28 & NA & 53.6 & 36.2 \\
F & 28 & 50.7 & 56.2 & 34.1 \\
M & 29 & 45.5 & 48.9 & 36.5 \\
F & 33 & 44.1 & 57.3 & 29.3 \\
F & 35 & 53.2 & 57.1 & 34.9 \\
F & 38 & 49.5 & 58.4 & 28.9 \\
F & 39 & 50.2 & 58.8 & 32.3 \\
M & 43 & 49.5 & 58.5 & 37.6 \\
\hline
\end{tabular}

Mean values are across left and right lateral rectus, inferior rectus, medial rectus, and superior rectus

$E O M$ extra-ocular muscle, $N A$ not available, ROEM range of eye movement individually (all $\mathrm{p}<0.01$ ). There was no significant quantitative difference in EOM cross-sectional area and T2 detected between the right and left orbit in either patients or controls, or between the CPEO and KSS subgroups, when muscles were compared both individually and collectively.

\section{Relationships between clinical and magnetic resonance imaging measures}

Correlations between clinical and MRI measures were assessed in the patient group (Fig. 3). There was a significant negative correlation between the patients' overall ROEM and the mean T2 relaxation time of the EOMs (rho $=-0.89, \mathrm{p}<$ 0.01). Mean EOM area did not correlate with disease duration or ROEM. Muscles graded as showing marked atrophy had significantly smaller relative cross sectional area (59\% of control value) than those graded as no (98\% control value) or mild ( $93 \%$ control value) atrophy $(\mathrm{p}<0.001)$. However, the difference in size between mild and no atrophy was not significant.

\section{Discussion}

We examined MRI EOM findings in a homogenous group of nine patients with CPEO caused by single mtDNA deletions compared with those in age- and gender-matched healthy controls. We describe differences in EOM radiological appearance, cross-sectional area, and T2 in CPEO patients compared to controls, and a significant negative correlation between 
Table 3 Mean range of eye movement, extra-ocular muscle cross-sectional area and T2 relaxation time in healthy control and patient groups

\begin{tabular}{|c|c|c|c|c|c|c|c|c|c|c|c|c|}
\hline & \multicolumn{5}{|c|}{ Control } & \multicolumn{5}{|c|}{ Patient } & \multicolumn{2}{|l|}{ Difference } \\
\hline & Mean & s.d. & $\mathrm{n}$ & Max & Min & Mean & s.d. & $\mathrm{n}$ & Max & Min & Mean $(95 \% \mathrm{CI})$ & p-value \\
\hline ROEM (degrees) & 49.3 & 3.0 & 8 & 53.2 & 44.1 & 13.3 & 8.2 & 8 & 25.8 & 3.1 & $-36.0(-42.6$ to -29.3$)$ & $<0.001$ \\
\hline Area $\left(\mathrm{mm}^{2}\right)$ & 33.6 & 3.1 & 9 & 37.6 & 28.9 & 19.2 & 3.9 & 9 & 25.0 & 13.8 & $-14.4(-17.9$ to -10.8$)$ & $<0.001$ \\
\hline $\mathrm{T} 2(\mathrm{~ms})$ & 55.1 & 4.4 & 9 & 46.9 & 58.8 & 77.8 & 7.0 & 9 & 67.8 & 85.7 & $-22.7(-28.4$ to -16.9$)$ & $<0.001$ \\
\hline
\end{tabular}

CI confidence interval, max maximum, min minimum, $n$ number, ROEM range of eye movement, s.d. standard deviation

EOM T2 and ROEM, suggesting MRI can provide functionally relevant measures of CPEO disease severity.

Earlier imaging studies have identified EOM involvement as characteristic of CPEO [4-7, 11]. However, these are generally in smaller, patient-only case series with genetically undetermined disease without quantitative assessment in all five EOMs. We have, for the first time, performed a systematic evaluation of EOM MRI signal changes in comparison with matched controls, and correlated MRI measurements of EOM size and condition with clinical functional measurements.

Previous reports of EOM atrophy in CPEO are mixed and rather limited: Carlow et al. [4] examined MR, LR and IR volume in eight clinically diagnosed CPEO patients showing an overall $41 \%$ reduction vs. controls. In a smaller series, EOM atrophy was reported on CT imaging of four clinically diagnosed CPEO patients [7]. Ortube et al. [6] detected isolated atrophy of SR and SO in five CPEO patients compared with controls. Finally, more recent work confirmed a primary myopathic rather than supranuclear pathway dysfunction aetiology for the limitation of eye movement in CPEO as evidenced by significant EOM atrophy in patients with single or multiple mtDNA deletions compared with controls [11]. Our EOM cross-sectional area results, incorporating SR and SO muscles in addition to MR, LR and IR, were consistent with these findings.

EOM atrophy is reported in a number of other neuromuscular disorders. Cranial nerve palsies (III, IV or VI) cause atrophy which is restricted to the muscle innervated by the affected nerve, thereby making it straight-forward to distinguish the affected muscle radiologically [6]. EOM atrophy is variably apparent in myasthenia gravis, but consistently seen in cases left untreated [7]. Radiological atrophy of clinically affected muscles has also been found in congenital fibrosis of EOMs type 1 [12] and type 2 [13] (now known to be dysinnervation syndromes). Taken together, these studies suggest that EOM atrophy is a feature of late-stage muscle pathology irrespective of the underlying cause, comparable to the atrophy of skeletal limb muscles on MRI in both neurogenic and myopathic disorders [14].

While EOM cross-sectional area has previously been investigated in CPEO, correlations between muscle dimensions and other quantitative MRI measures, and clinical functional

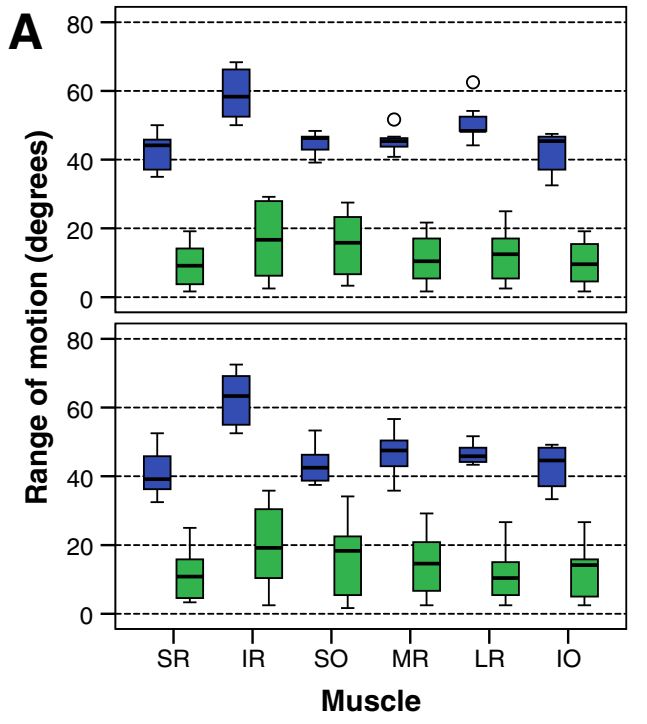

Fig. 1 Box plot of control (blue) and patient (green) values in each extraocular muscle: range of motion (A), cross-sectional area (B) and T2 relaxation time $(\mathbf{C})$. The box represents 25 th -75 th percentiles, the solid line in the box represents the median value, stems are to maximum and
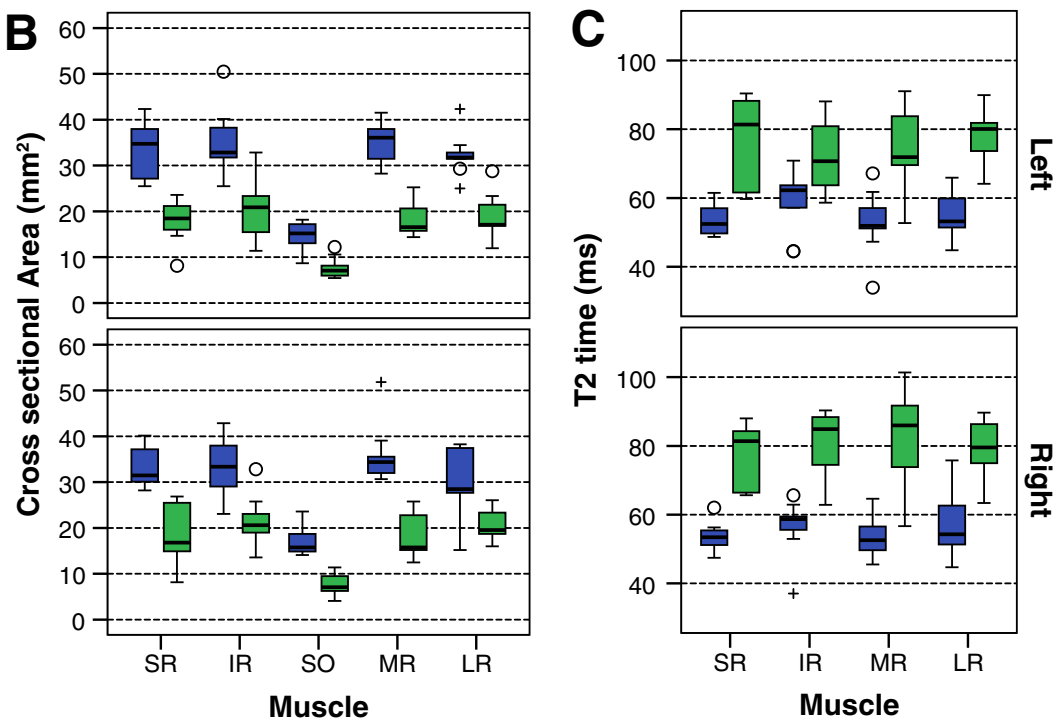

minimum values, $\mathrm{o}=$ minor outlier; $+=$ major outlier. $I O$ inferior oblique, $I R$ inferior rectus, $L R$ lateral rectus, $M R$ medial rectus, $S O$ superior oblique, $S R$ superior rectus 

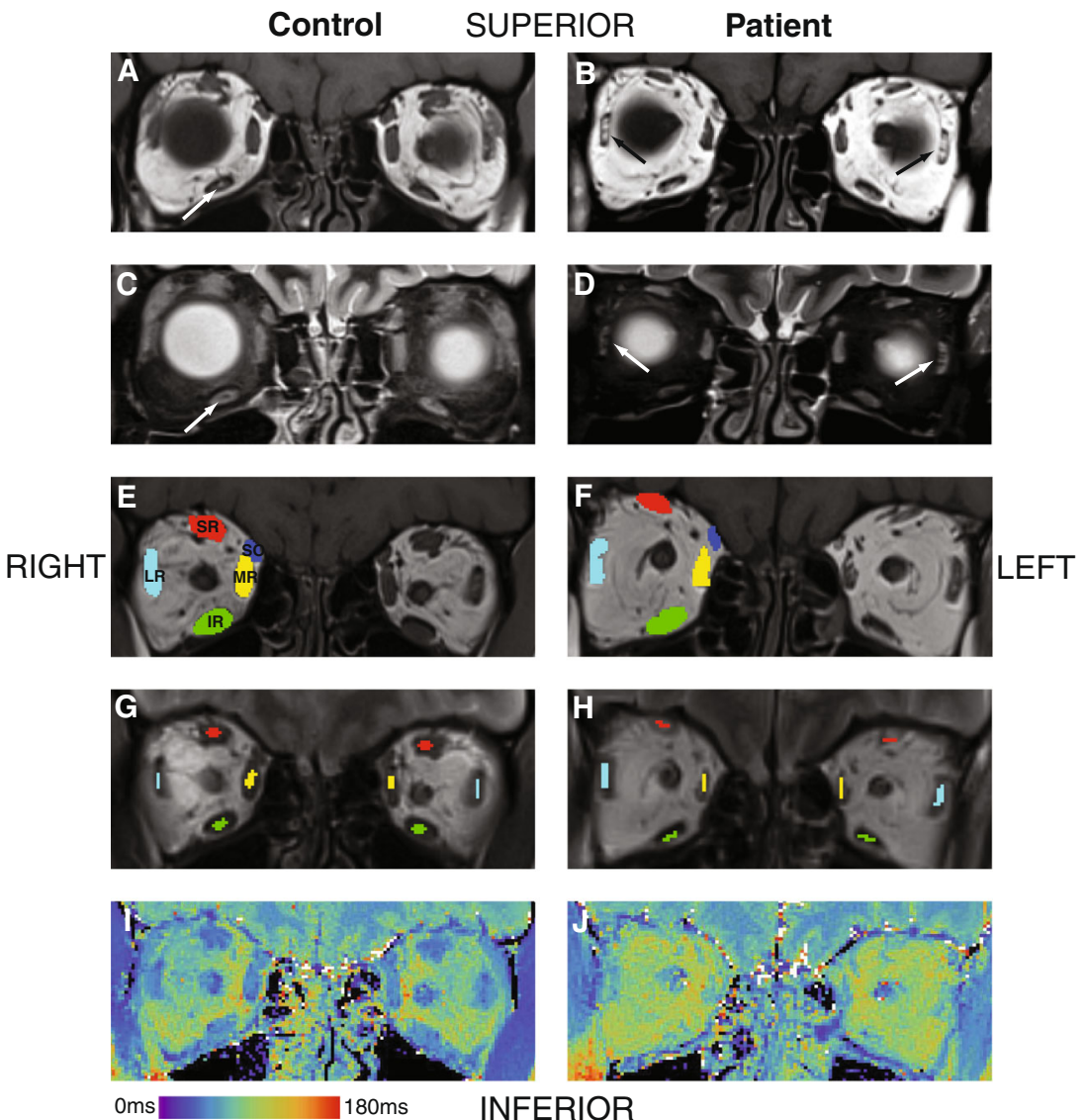

Fig. 2 MRI of the orbits in the coronal plane displaying a healthy control on the left column (panels A, C, E, G, I) and a patient with chronic progressive external ophthalmoplegia (CPEO) on the right column (panels B, D, F, H, J). Coronal T1-weighted (panels A and B) and corresponding short-tau inversion recovery (STIR, panels $\mathbf{C}$ and $\mathbf{D}$ ) sequences demonstrate T1-weighted hyperintense and STIR-suppressed foci intrinsic to the extra-ocular muscles (EOMs). If the diameter of the focus was approximately equal in all dimensions in the coronal plane it was termed a 'dot', whilst if unequal it was termed a 'streak'. An example of a 'dot' is demonstrated in the right inferior rectus muscle on paired panels $\mathrm{A}$ and $\mathrm{C}$, and examples of 'streaks' are shown in both lateral recti on paired panels

Table 4 Frequency of T1-weighted magnetic resonance imaging changes in healthy control and patient extra-ocular muscles

\begin{tabular}{lrrrrrr}
\hline & Controls & \multicolumn{5}{c}{ Patients } \\
\hline Muscle & None & Dot & Streak & None & Dot & Streak \\
SR & 16 & 2 & 0 & 7 & 0 & 11 \\
IR & 4 & 2 & 12 & 8 & 0 & 10 \\
SO & 0 & 14 & 4 & 4 & 6 & 8 \\
MR & 8 & 4 & 6 & 0 & 0 & 18 \\
LR & 13 & 2 & 3 & 3 & 2 & 13 \\
IO & 18 & 0 & 0 & 14 & 1 & 3 \\
Total & 59 & 24 & 25 & 36 & 9 & 63 \\
\hline
\end{tabular}

Left and right eye combined

$I O$ inferior oblique, $I R$ inferior rectus, $L R$ lateral rectus, $M R$ medial rectus, $S O$ superior oblique, $S R$ superior rectus
B and D respectively (arrows). Panels $\mathbf{E}$ and $\mathbf{F}$ demonstrate an example of the regions of interest (ROI) used to calculate the surface area of each of the EOMs within the right orbit in a control volunteer and CPEO patient respectively: superior-rectus levator complex (SR); superior oblique (SO); medial rectus (MR); lateral rectus (LR); and inferior rectus (IR). Central ROI were placed within the same muscles for the T2-relaxometry measurements in order to avoid contamination by the surrounding fat, as demonstrated in panels $\mathbf{G}$ and $\mathbf{H}$ within the right orbits of a control volunteer and CPEO patient respectively. Panels $\mathbf{I}$ and $\mathbf{J}$ demonstrate false colour T2-relaxometry maps

measurements, have not been reported. Such correlations, if observed, would support the validity of MRI measures as biologically relevant disease indices. Although we found no correlation between muscle atrophy and reduced ROEM or disease duration, there was a significant negative correlation between T2 and ROEM in the patients. This indicates that although measures relating to EOM volume may not be useful to quantify severity in patients with long-established disease, measures such as EOM T2, reflecting variations in muscle composition rather than size, may provide valid alternative functionally relevant markers. Future studies will be required to establish the sensitivity of EOM T2 to longitudinal change, and to establish the sensitivity of the measure to partial volume confounds due to surrounding intra-orbital fat.

In addition to atrophic changes, we also detected EOM MRI signal abnormalities in patients with CPEO, apparent qualitatively as an increased frequency of $\mathrm{T} 1 \mathrm{w}$ hyperintense 
A
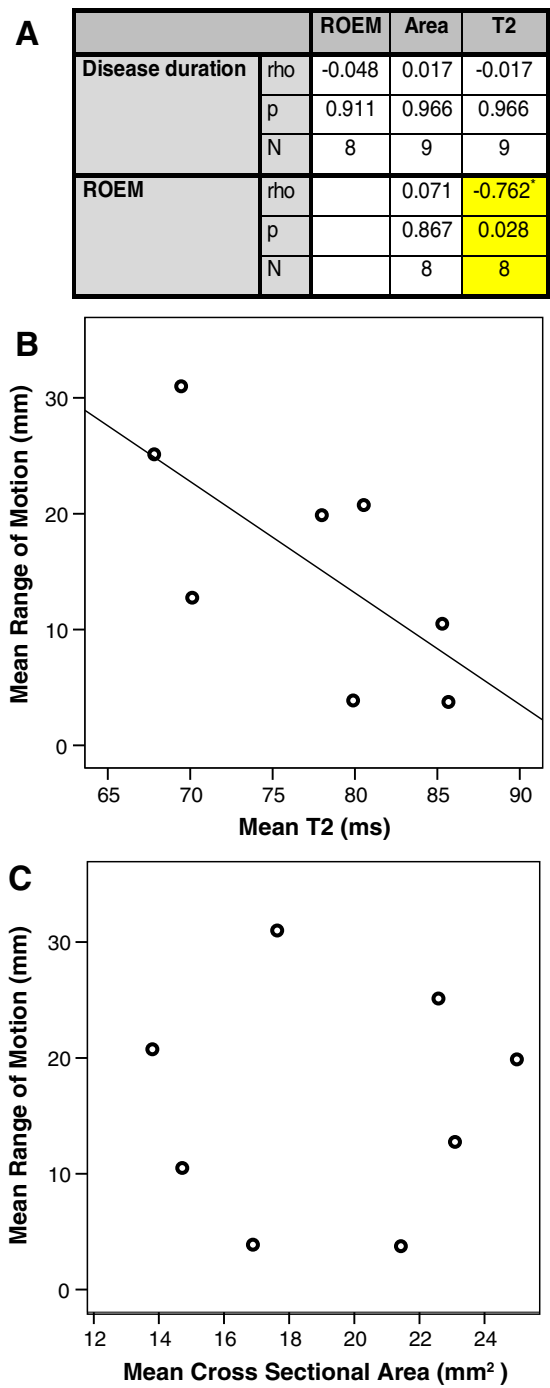

Fig. 3 Spearman correlations between clinical and quantitative MRI measures in patients with chronic progressive external ophthalmoplegia (A). A single mean value for each parameter was calculated in each patient for the common regions of interest (superior rectus, inferior rectus, medial rectus and lateral rectus). Range of eye movement (ROEM) correlated with $\mathrm{T} 2$ relaxation time. The cross-sectional area did not correlate with either ROEM or disease duration. Scatter plots are shown for T2 versus ROEM (B) and area versus ROEM $(\mathbf{C})$

streaks with very occasional STIR hyperintensity. Central streaks of EOM hyperintensity on T1w MRI have previously been reported in CPEO [6], although we also saw similar appearances in some of the control EOMs. While the frequency of these hyperintensities was greater in the patient group, their presence in the healthy control images suggests such changes are not specific markers of CPEO pathology. Similar changes have also been shown to occur with muscle atrophy in patients with congenital fibrosis of the EOMs type 1 [12].

This is the first study to measure EOM T2 in a genetically homogenous CPEO cohort. While acute inflammation prolongs $\mathrm{T} 2$, and is used to quantify EOM inflammation in thyroid-related ophthalmopathy [15-19], inflammation is unlikely to be a pathological substrate of EOM T2 change in $\mathrm{CPEO}$, since inflammatory infiltrates are usually absent in the muscle biopsies of patients with mitochondrial disease. This is supported by the predominant absence of EOM STIR hyperintensities in our patient images, and suggests increased intramuscular lipid as the source of T2 elevation since our T2 relaxometry acquisition was not fat-suppressed. A further analysis (results not shown) that attempted to fit a bi-exponential model to the multi-echo decay data was not sufficient to obtain putative lipid and muscle-water components with independent $\mathrm{T} 2$ values, and more sophisticated future measurements will be required to unambiguously establish the origin of the total muscle T2 elevation. A further caveat to interpretation of the T2 findings is the potential susceptibility of the measurements to contamination from the surrounding fatty substrate, despite our precautions in defining conservative ROI boundaries. Nevertheless, elevated patient EOM T2 compared with controls was consistent and, coupled with the correlation observed between EOM T2 and ROEM measures, should motivate future more sophisticated investigations.

Accurate, valid and reliable biomarkers of disease progression are lacking in mitochondrial disease but will be required to assess the efficacy of novel therapies in treatment trials. We have demonstrated the feasibility of quantitative MRI to provide measures reflecting disease severity in individual EOMs in patients with CPEO. While EOM atrophy is a clear radiological feature, its uniformity in patients and lack of correlation with ROEM suggests it might not provide a meaningful measure of progression in patients studied a significant time after disease onset. In contrast, EOM T2 prolongation was associated with cross-sectional variations in ROEM in our study group, and so may represent a useful biomarker for future clinical trials involving patients with similar disease durations. Future investigations will evaluate the added value of combining T2 measurement with the technically challenging but promising approach of EOM quantitative diffusion weighted imaging [19].

In conclusion, we have examined the EOM MRI appearance of patients with CPEO due to single mtDNA deletions and demonstrated atrophy of all EOMs, more frequent $\mathrm{T} 1 \mathrm{w}$ hyperintensities in patients than controls and uniform prolongation of EOM T2 in patients. A correlation between EOM T2 and reduced ocular excursion facility suggests that this parameter might provide a valid, clinically relevant, objective measure of disease severity useful to monitor patients with established CPEO with possible value in future clinical trials.

Acknowledgments The scientific guarantor of this publication is Robert D.S. Pitceathly (R.D.S.P.). The authors of this manuscript declare no relationships with any companies whose products or services may be related to the subject matter of the article. No complex statistical methods were necessary for this paper. Institutional Review Board approval was obtained. Written informed consent was obtained from all subjects (patients and healthy controls) in this study. 
R.D.S.P. is funded by MRC grant number G0800674. M.G.H., J.M.M. and C.D.J.S. are supported by an MRC Centre grant G0601943. S.R. is supported by Great Ormond Street Hospital Children's Charity. This study was supported by the NIHR UCLH/UCL Comprehensive Biomedical Research Centre and undertaken at University College London Hospitals/University College London, which received a proportion of funding from the Department of Health's National Institute for Health Research Biomedical Research Centres funding scheme. Methodology: prospective, observational, performed at one institution.

Open Access This article is distributed under the terms of the Creative Commons Attribution-NonCommercial 4.0 International License (http:// creativecommons.org/licenses/by-nc/4.0/), which permits any noncommercial use, distribution, and reproduction in any medium, provided you give appropriate credit to the original author(s) and the source, provide a link to the Creative Commons license, and indicate if changes were made.

\section{References}

1. Pfeffer G, Majamaa K, Turnbull DM, Thorburn D, Chinnery PF (2012) Treatment for mitochondrial disorders. Cochrane Database Syst Rev 4, CD004426

2. Pitceathly RDS, McFarland R (2014) Mitochondrial myopathies in adults and children: management and therapy development. Curr Opin Neurol 27(5):576-82

3. Schaefer AM, McFarland R, Blakely EL, He L, Whittaker RG, Taylor RW et al (2008) Prevalence of mitochondrial DNA disease in adults. Ann Neurol 63(1):35-39

4. Carlow TJ, Depper MH, Orrison WW (1998) MR of extraocular muscles in chronic progressive external ophthalmoplegia. AJNR Am J Neuroradiol 19(1):95-99

5. Peters S, Vorgerd M, Heyer CM (2006) Chronic progressive external ophthalmoplegia plus: diagnosis with muscular magnetic resonance tomography. Röfo 178(10):1030-1032

6. Ortube MC, Bhola R, Demer JL (2006) Orbital magnetic resonance imaging of extraocular muscles in chronic progressive external ophthalmoplegia: specific diagnostic findings. J AAPOS 10(5): 414-418

7. Okamoto K, Ito J, Tokiguchi S, Furusawa T (1996) Atrophy of bilateral extraocular muscles. CT and clinical features of seven patients. J Neuroophthalmol 16(4):286-288
8. Richardson C, Smith T, Schaefer A, Turnbull D, Griffiths P (2005) Ocular motility findings in chronic progressive external ophthalmoplegia. Eye (Lond) 19(3):258-263

9. Haggerty H, Richardson S, Mitchell KW, Dickinson AJ (2005) A modified method for measuring uniocular fields of fixation: reliability in healthy subjects and in patients with Graves orbitopathy. Arch Ophthalmol 123(3):356-362

10. Yushkevich PA, Piven J, Hazlett HC, Smith RG, Ho S, Gee JC et al (2006) User-guided 3D active contour segmentation of anatomical structures: significantly improved efficiency and reliability. Neuroimage 31(3):1116-1128

11. Yu-Wai-Man C, Smith FE, Firbank MJ, Guthrie G, Guthrie S, Gorman GS et al (2013) Extraocular muscle atrophy and central nervous system involvement in chronic progressive external ophthalmoplegia. PLoS ONE 8(9), e75048

12. Demer JL, Clark RA, Engle EC (2005) Magnetic resonance imaging evidence for widespread orbital dysinnervation in congenital fibrosis of extraocular muscles due to mutations in KIF21A. Invest Ophthalmol Vis Sci 46(2):530-539

13. Bosley TM, Oystreck DT, Robertson RL, al Awad A, Abu-Amero K, Engle EC (2006) Neurological features of congenital fibrosis of the extraocular muscles type 2 with mutations in PHOX2A. Brain 129(Pt 9):2363-2374

14. Wattjes MP, Kley RA, Fischer D (2010) Neuromuscular imaging in inherited muscle diseases. Eur Radiol 20(10):2447-2460

15. Nagy EV, Toth J, Kaldi I, Damjanovich J, Mezosi E, Lenkey A et al (2000) Graves' ophthalmopathy: eye muscle involvement in patients with diplopia. Eur J Endocrinol 142(6):591-597

16. Pauleit D, Schüller H, Textor J, Leutner C, Keller E, Sommer T et al (1997) MR relaxation time measurements with and without selective fat suppression (SPIR) in endocrine orbitopathy. Röfo 167(6): $557-564$

17. Ulmer JL, Logani SC, Mark LP, Hamilton CA, Prost RW, Garman JN (1998) Near-resonance saturation pulse imaging of the extraocular muscles in thyroid-related ophthalmopathy. AJNR Am J Neuroradiol 19(5):943-950

18. Utech CI, Khatibnia U, Winter PF, Wulle KG (1995) MR T2 relaxation time for the assessment of retrobulbar inflammation in Graves' ophthalmopathy. Thyroid 5(3):185-193

19. Politi LS, Godi C, Cammarata G, Ambrosi A, Iadanza A, Lanzi R et al (2014) Magnetic resonance imaging with diffusion-weighted imaging in the evaluation of thyroid-associated orbitopathy: getting below the tip of the iceberg. Eur Radiol 24(5):1118-1126 